\title{
GRAPHICAL AND STATISTICAL ANALYSIS OF AIRPLANE PASSENGER CABIN RF COUPLING PATHS TO AVIONICS
}

\author{
Madiha Jafri, Old Dominion University, Norfolk, Virginia \\ Jay Ely, NASA Langley Research Center, Hampton, Virginia \\ Dr. Linda Vahala, Old Dominion University, Norfolk, Virginia
}

\begin{abstract}
:
Portable wireless technology provides many benefits to modern day travelers. Over the years however, numerous reports have cited portable electronic devices (PEDs) as a possible cause of electromagnetic interference (EMI) to aircraft navigation and communication radio systems. PEDs may act as transmitters, both intentional and unintentional, and their signals may be detected by the various radio receiver antennas installed on the aircraft. Measurement of the radiated field coupling between passenger cabin locations and aircraft communication and navigation receivers, via their antennas is defined herein as interference path loss (IPL). IPL data is required for assessing the threat of PEDs to aircraft radios, and is very dependent upon airplane size, the interfering transmitter position within the airplane, and the location of the particular antenna for the aircraft system of concern. NASA Langley Research Center, Eagles Wings Inc., and United Airlines personnel performed extensive IPL measurements on several Boeing 737 airplanes.
\end{abstract}

This paper provides a graphical and statistical analysis of IPL data measured onboard two Boeing 737 airplanes. The analysis reveals valuable insight into EMI field propagation characteristics, measurement repeatability, selection of test equipment, and interpretation of measurement data related to IPL.

\section{Introduction:}

In the Spring of 2002, engineers from NASA Langley Research Center, United Airlines, and Eagles Wings Inc. (EWI) teamed to collect extensive IPL data on several out-of-service Boeing 737-200 and 747-400 airplanes. Systems considered were the instrument landing system Glideslope (GS), Traffic Alert and Collision Avoidance System (TCAS), VHF Communication System, instrument landing system Localizer (LOC) and VHF Omniranging (VOR) system. [1] One goal was to investigate measurement repeatability when acquiring IPL data on multiple identical airplanes, using different instrumentation and different test teams. Another goal was to acquire detailed IPL data from seat and aisle locations, in addition to the

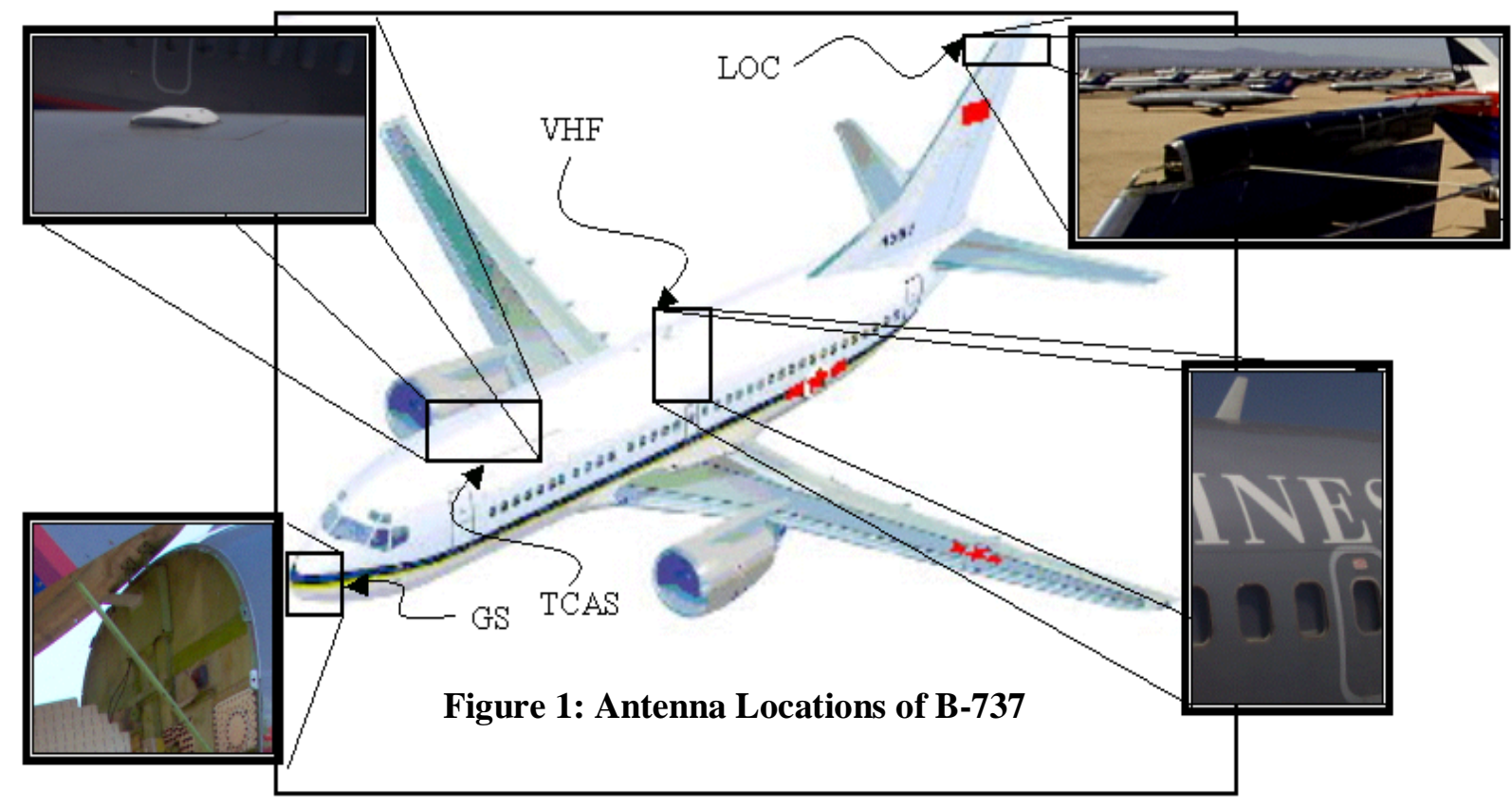


more typically performed window locations. Proper analysis of this data was expected to provide insight into the measurement uncertainty of more typical IPL data collected on a single representative airplane, with a limited set of measurement locations.

This paper is divided into five major sections. Section 1 provides background for understanding the Interference Path Loss measurement process as used for analysis herein. Section 2 provides a graphical analysis of IPL electromagnetic field patterns at every seat and window location of two different Boeing 737-200 airplanes, using MATLAB. The graphs from one set of airplane data are compared to another to evaluate repeatability of the IPL measurements. Section 3 provides probability distribution plots for a quantitative comparison of range, mean, standard deviation and variance of IPL measurement data. Section 4 extends the graphical and statistical analysis to a window-only subset of the overall IPL data set. This allows a comparison of statistical parameters as they would be calculated from more commonly available IPL measurement datasets. Section 5 provides a comparison of two types of test antennas, biconical and dipole, to document the effect on IPL measurements and patterns due to different test antennas.

\section{IPL Measurements:}

Before attempting to understand the analysis of IPL data, it is necessary to review how the data was measured. IPL, as addressed herein, is particularly focused upon in-band, on-channel type EMI to aircraft radios, via their antennas. This does not include EMI to aircraft radios outside their radio frequency (RF) passband, and does not include radiated field (or conducted) coupling to wiring and equipment apertures.

IPL data was taken by radiating a low powered continuous wave $(\mathrm{CW})$ test signal, frequencysynchronized to the spectrum analyzer sweep and fed to the test transmitting Antenna via a doubleshielded RF cable. The spectrum analyzer, laptop computer controller, signal generators, RF Amplifiers and preamplifiers were located inside the aircraft. The spectrum analyzer input cable was connected to the aircraft radio receiver rack cable in the avionics equipment bay.
To perform an IPL measurement, the team measured the RF power loss between the calibrated signal source and a spectrum analyzer, via the entire length of test cables plus the aircraft cable, plus the free space loss between the reference antenna and the aircraft antenna. Figure 2 provides a description of the test setup. To obtain a calibrated IPL measurement, test cable losses were measured separately by connecting ends (1) and (2) together, and subtracting this loss, in $\mathrm{dB}$, from the raw measurement. In some cases, the aircraft cable loss could also be measured by accessing end (3), but this data is not relevant to the analysis reported herein.

Individual IPL measurements were obtained by moving the test antenna to numerous seat, window and aisle locations throughout the airplane. A complete description of the measurement process may be found in [2].

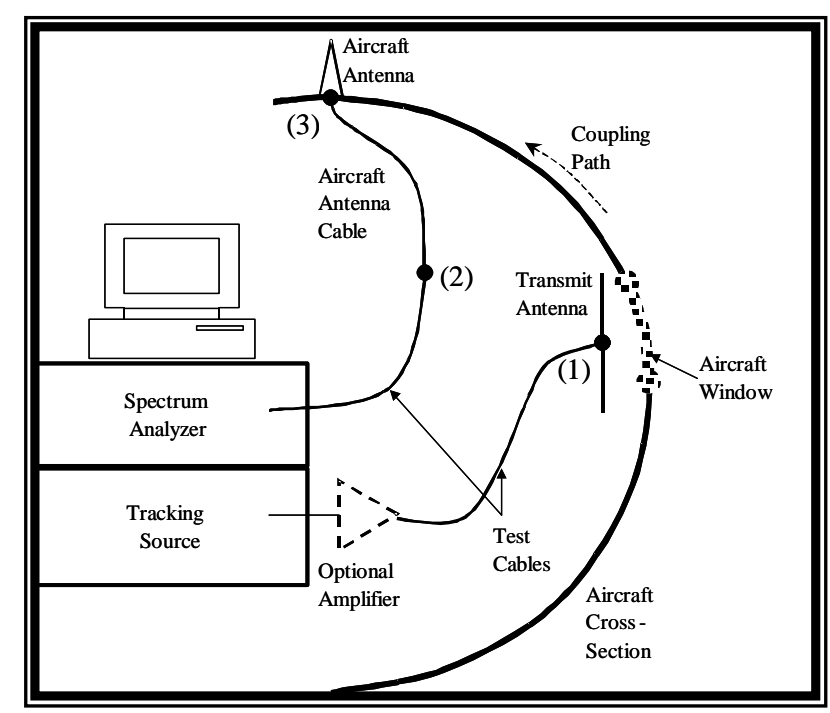

Figure 2: Description of Test Setup

\section{Graphical Representation of Airline Passenger Cabin RF Coupling paths to Avionics:}

Using the method described in Section 1, IPL measurements were taken at each row of the aircraft, not only at each window and seat locations, but also in between seat locations and in the aisle. Thus, a total of eight measurements were taken at each row of the aircraft's seating, represented in 
Figure 3 as $\mathrm{W}, \mathrm{A}, \mathrm{AB}, \mathrm{B}, \mathrm{BC}, \mathrm{C}, \mathrm{CI}$, and I, where "W" represents "Window" location and "I" represents "Aisle".

At each location, measurements were taken with the antenna in vertical as well as horizontal positions. Dual polarization IPL measurements double the amount of test time and resulting data, but reveal distinctly different IPL characteristics related to aircraft configuration and aircraft antenna polarization.

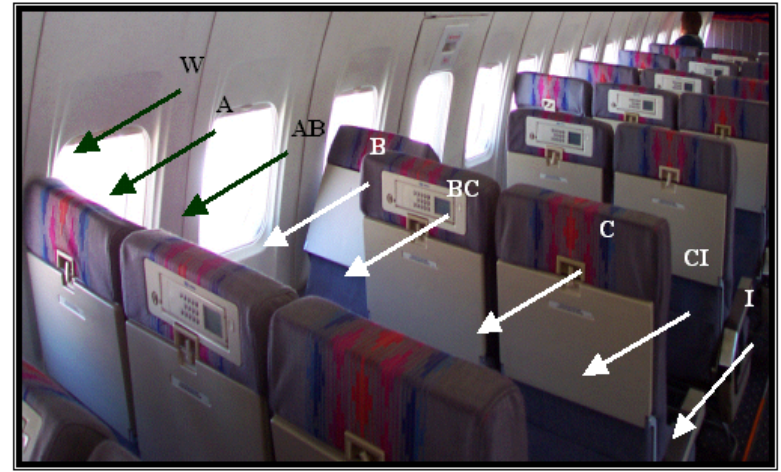

Figure 3: Test Locations of Each Aircraft Row

A coloring scheme was then applied to the graphs so that the red regions indicate the greater coupling (or the lower path loss) while the blue regions indicate the lower coupling (or the higher path loss). However, for the purpose of better visibility, it was essential to rotate the $3-\mathrm{D}$ graphs into 2-D and use the color map to represent the peaks and valleys of the z-axis. Figures 4, 5, 6 and 7 show each aircraft system antenna location and the estimated region of greatest coupling within the aircraft passenger cabin (2-D representation). The schematic is followed by the calibrated visual representation of the IPL, both in horizontal and vertical polarizations.

\section{GS Analysis:}

The estimated and the measured result of the GS IPL pattern are shown in Figure 4. Since the GS Antenna is located in the nose of the aircraft, it was predicted that the greatest coupling would occur at the earlier rows of the aircraft as well (represented by the red region on the schematic). It was estimated that the greatest coupling would occur near the first exit door of the aircraft.

Graphical results confirm that the lowest IPL was indeed in the front of the aircraft. Because the aircraft GS antenna is horizontally polarized, lower horizontal IPL values were expected. According to the graphs, better coupling does indeed occur between the test antenna and aircraft antenna when the test antenna is horizontally polarized.

\section{TCAS Analysis:}

The estimated and the measured result of the TCAS IPL pattern are shown in Figure 5. Since the TCAS Antenna is located on top of the second window from the front of the aircraft, it was

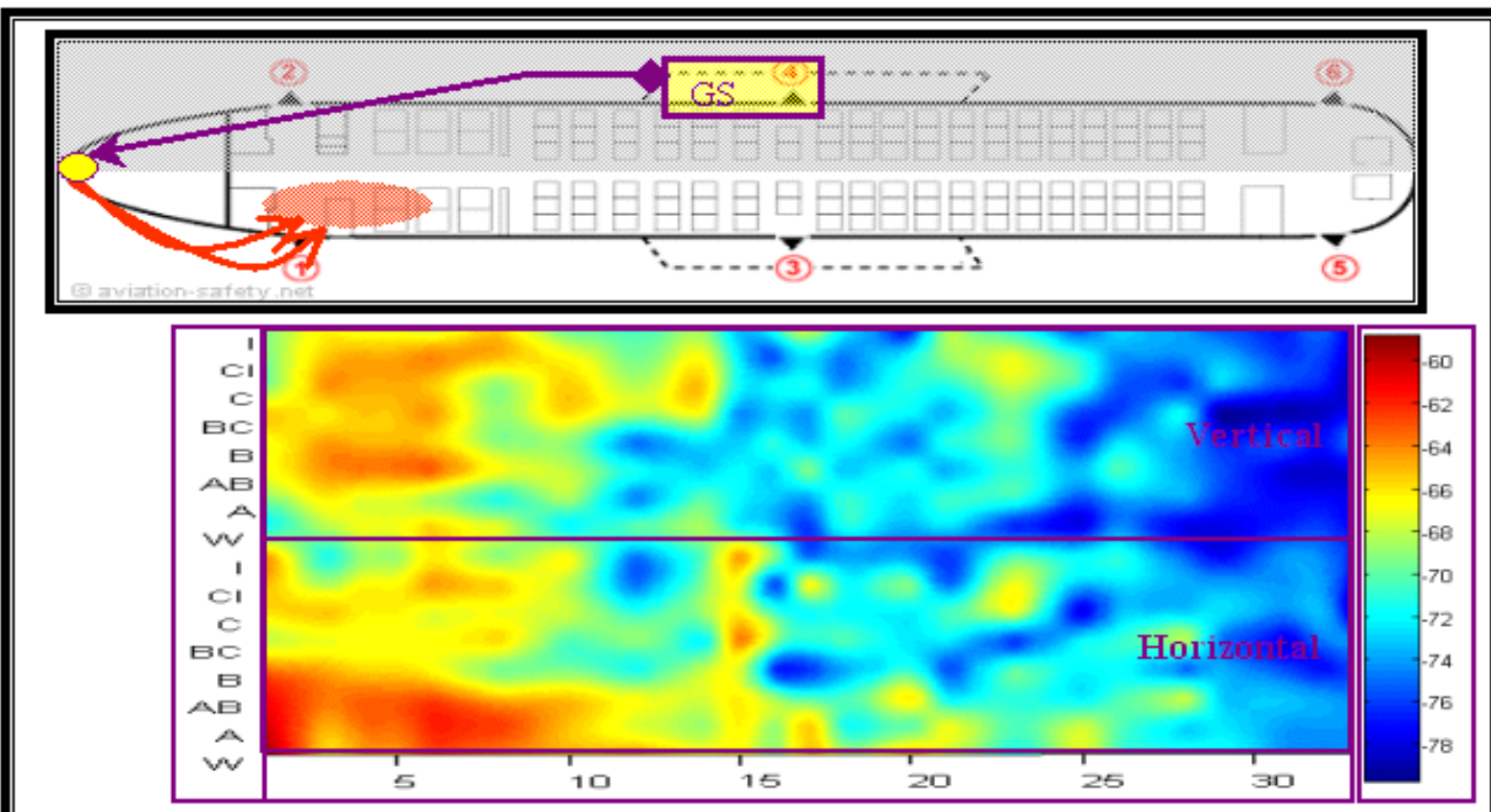

Figure 4: GS Prediction and Actual Results 
predicted that the greatest coupling would occur near the front exit of the aircraft, as well as near the second window location.

Graphical results confirm that the lowest IPL was indeed observed at these locations. Because the aircraft TCAS antenna is vertically polarized, lower vertical IPL values were expected at optimal coupling locations. According to the graphs, better coupling does indeed occur between the test antenna and aircraft antenna when the test antenna is vertically polarized.

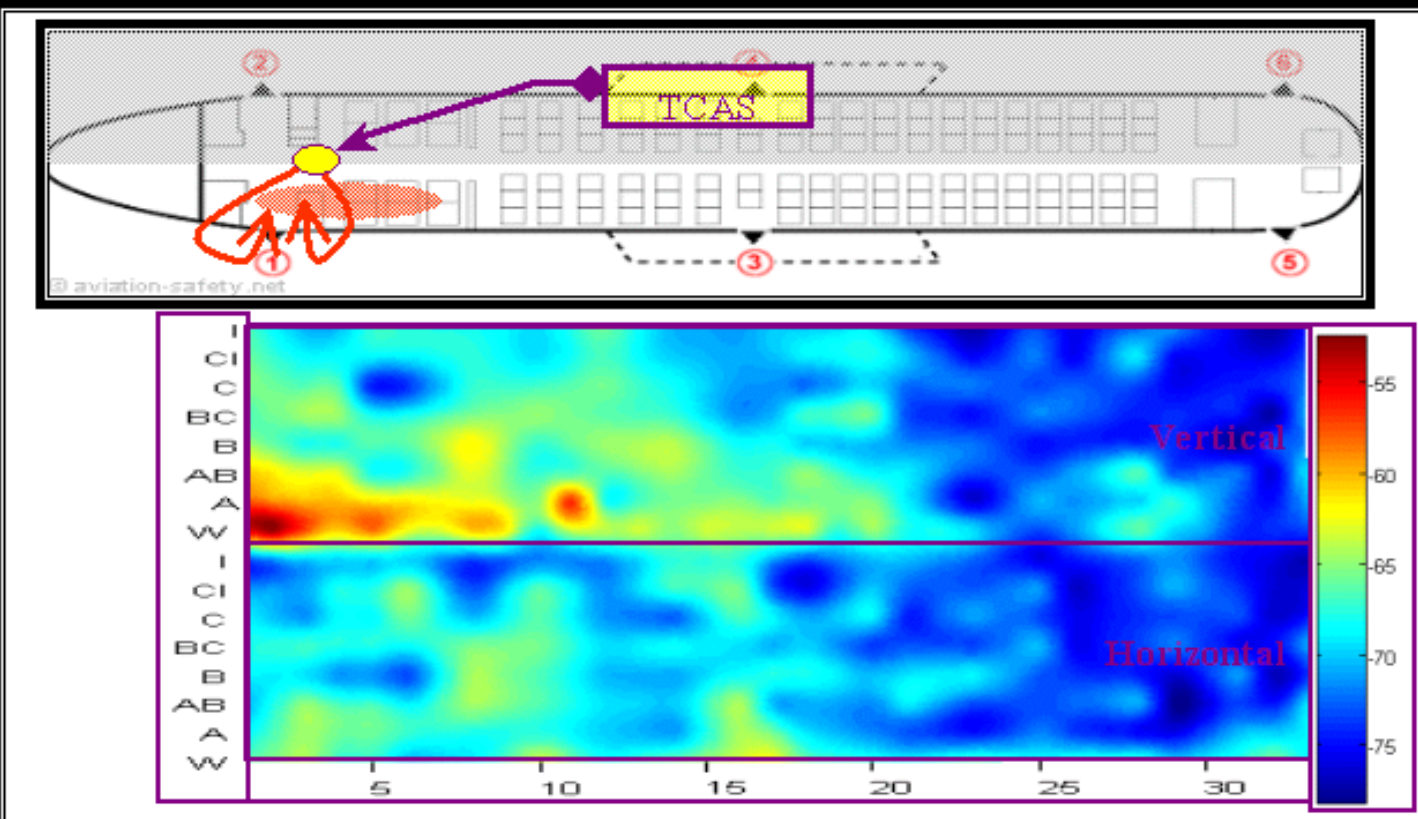

Figure 5: TCAS Prediction and Actual Results
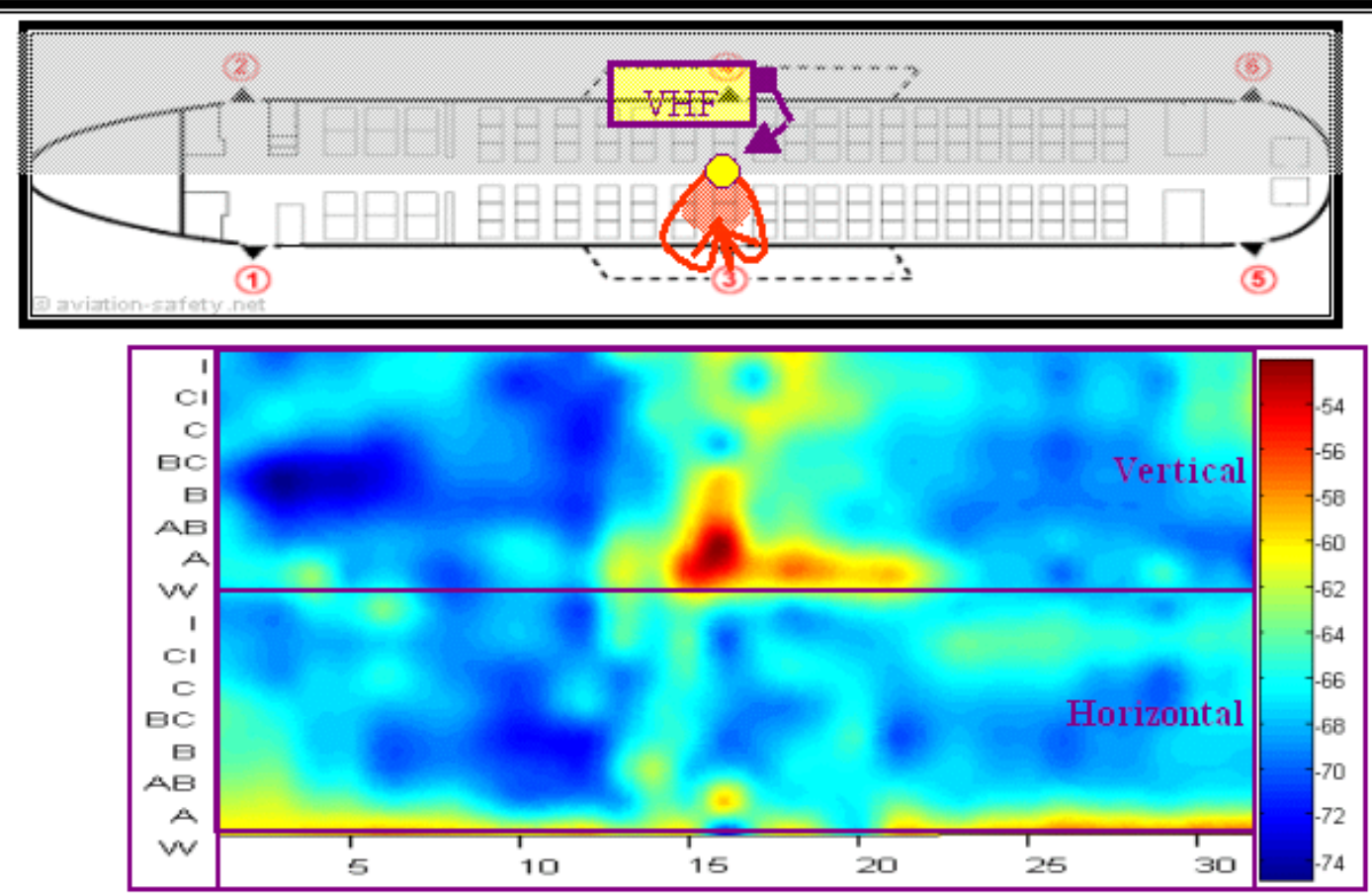

Figure 6: VHF Prediction and Actual Results 


\section{VHF Com. Analysis:}

The estimated and the measured result of the VHF Com. IPL pattern are shown in Figure 6. The VHF Com. Antenna is located on top of the middle Emergency exit, precisely, window 16 of the B-737 airplane. Therefore, it was predicted that the greatest coupling would occur near window 16 (emergency exit). Also, since the aircraft antenna is vertically polarized, it was assumed that waves would couple better vertically than horizontally. Graphical results confirm that the vertical polarization is dominant and the greatest coupling occurs near the emergency exit, or window location 16 of the aircraft.

\section{LOC Analysis:}

The estimated and the measured result of the LOC IPL pattern are shown in Figure 7. The LOC antenna is located at the tip of the tail of the aircraft, and is horizontally polarized. Therefore, it was predicted that the greatest coupling would occur near the rear exit of the aircraft, with the test antenna in the horizontal polarization. Graphical results confirm that the horizontal polarization is dominant, however the greatest coupling does not occur toward the rear of the airplane.

The graphical plot indicates that the L2 doorway (rear left) is not in an optimal location to allow coupling to the back of the airplane, but the over-wing exit (at window 16) is.

\section{Comparison Data From Another Airplane:}

All graphical IPL data presented so far was obtained on United Airlines B737-200, nose number 1989. Another, identical set of data was obtained using a different airplane (United Airlines B-737 \#1997), a different set of instrumentation and cables, and a different test team. Again, for \#1997, all windows, seats, aisles, and in between seat and aisle data was taken. Figure 8 shows the graphical data for the GS, TCAS and VHF results for B-737 \#1997.

Comparing graphs in Figure 8.a, 8.b and 8.c with Figures 4, 5 and 6, many similarities in the patterns can be observed. Most importantly, the color regions and color scale magnitudes are very similar for both the \#1989 and \#1997 graphs. This assures that the IPL findings were similar, regardless of the different airplanes, test equipment and personnel performing the measurements. Full seat window and aisle data was not obtained for the LOC system on \#1997 due to excessive aircraft antenna/cable loss, therefore, that comparison could not be made in this analysis.

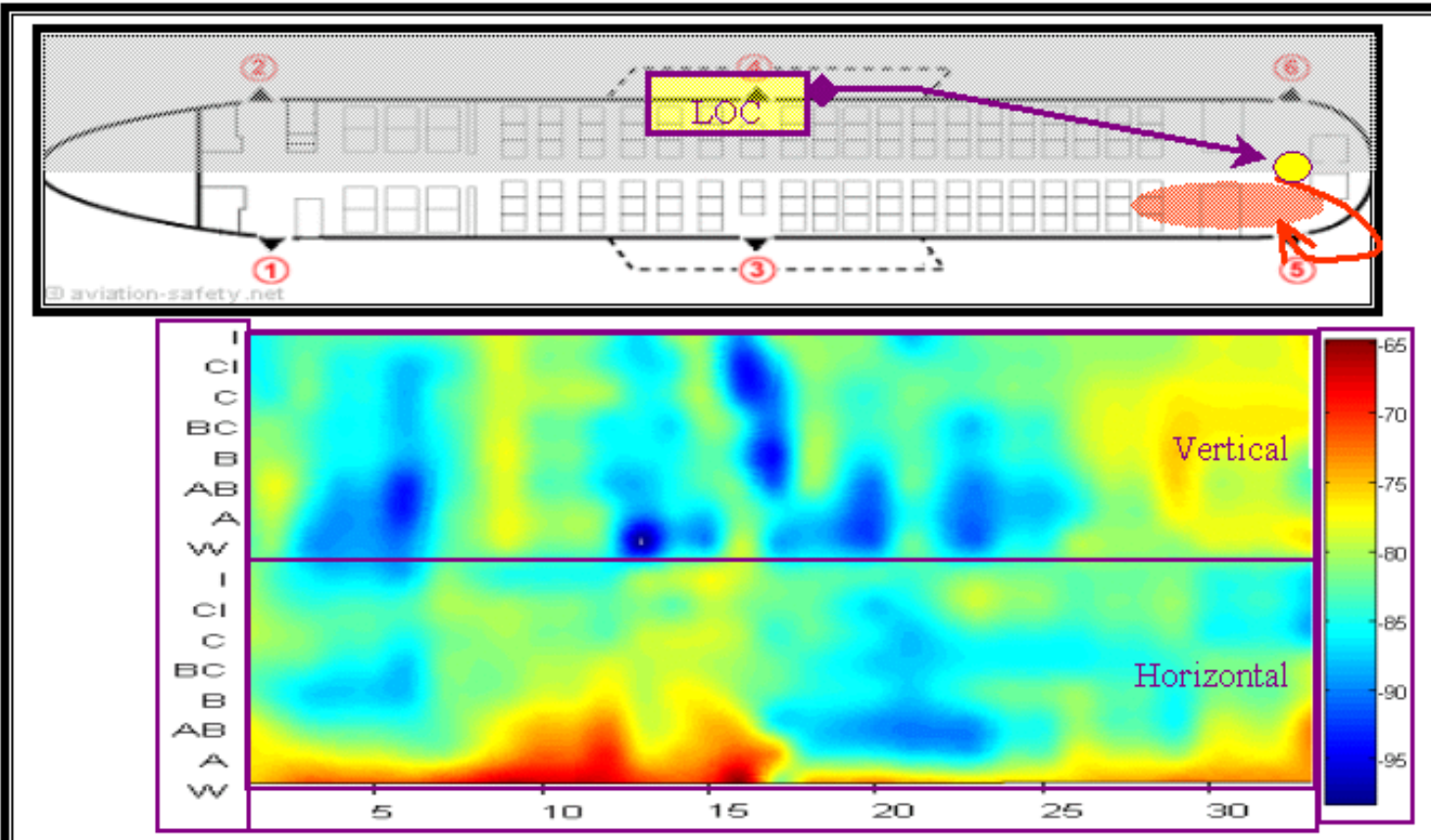

Figure 7: LOC Prediction and Actual Results 


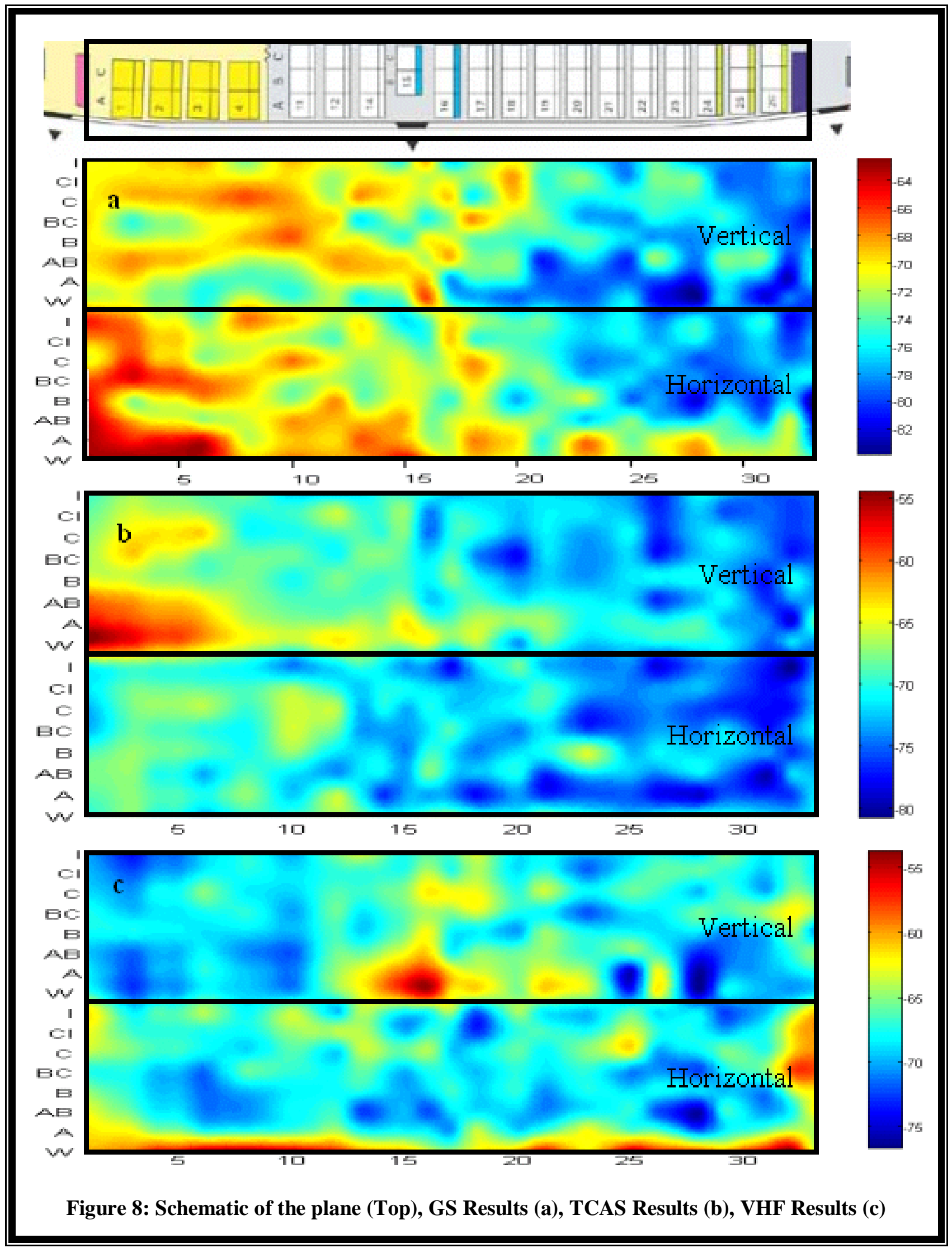




\section{Statistical Analysis of IPL:}

RTCA/DO-233 reported some IPL measurement data with minimums, averages, number of data points and standard deviations. [3] These quantitative statistical metrics allow tabular comparison of IPL data. The data used in the visual analysis in the previous section was transferred into normalized plots so that statistical metrics can be visualized, compared and assessed in tabular form.

In Figure 9, measurement data for each aircraft system and each polarization is plotted on the $\mathrm{x}$-axis while the $y$-axis contains the raw number of measurement locations on the airplane with a particular IPL value. The graphs on the left are for horizontal test antenna polarization, while the graphs on the right are results for vertical test antenna polarization. On the top right hand corner of each graph, the histogram's mean, standard deviation and variance are shown. Each plot axis is scaled the same to facilitate comparison of polarizations and IPL measurement distributions. (ie. $X$ axis IPL value ranges from 50 to $100 \mathrm{~dB}$, and $\mathrm{Y}$ axis number of measurements ranges from 0 to 35.) These distribution plots provide quantitative comparisons of the data, while revealing minimums, maximums and distribution trends not evident in numerical metrics.

Each distribution plot is accompanied by a best-fit normal statistical data distribution curve. Probability distribution analysis of IPL data will be the subject of subsequent work.

\section{GS Measurement Distribution Plot:}

In the graphical analysis of the GS data (see Figure 4), horizontal polarization coupling was shown to be dominant (meaning lower path loss). The aircraft GS antenna is horizontally polarized. The measurement distribution plot in Figure 9 shows the same GS data, with horizontal test antenna polarization having a slightly lower mean, higher standard deviation and therefore, higher variance than the vertical test antenna polarization. Most importantly, it can be seen that a few horizontally polarized test antenna measurements clearly define the minimum IPL locations.

\section{TCAS Measurement Distribution Plot:}

In the graphical analysis of the TCAS data (see Figure 5), the vertical polarization coupling was shown to be dominant. The aircraft TCAS antenna is vertically polarized. The measurement distribution plot in Figure 9 shows the same TCAS data, with vertical test antenna polarization having a slightly lower mean, higher standard deviation and therefore, higher variance than the horizontal test antenna polarization. Similar to GS (but with different polarization), it can be seen that a few vertically polarized test antenna measurements clearly define the minimum IPL locations.

\section{VHF Measurement Distribution Plot:}

In the graphical analysis of the VHF Com. data (see Figure 6), measurements revealed optimal coupling locations (minimum IPL) for both vertical and horizontal test antenna polarizations. Similarly, in Figure 9, the variance, mean and standard deviation of the horizontal and vertical measurement data are very close to each other. The mean differs by only 0.01 . However, the vertical polarization data has the (slightly) lower mean, and the higher standard deviation and variance leading to the conclusion that the test antenna couples slightly better to the VHF Com. system when it is in Vertical polarization. The aircraft VHF Com. antenna is vertically polarized. Again, it can also be seen that a few test antenna measurements clearly define the minimum IPL locations.

\section{LOC Measurement Distribution Plot:}

In the graphical analysis of the LOC data (see Figure 7), horizontal polarization coupling was shown to be dominant. The aircraft LOC antenna is horizontally polarized. The measurement distribution plot in Figure 9 shows distinct differences in the measurement data. The horizontal test antenna polarization data clearly has the lower mean, greater standard deviation and greater variance. The minimum IPL measurement is $10 \mathrm{~dB}$ lower for horizontal test antenna polarization than for vertical polarization, and dominated by several specific measurement locations. 


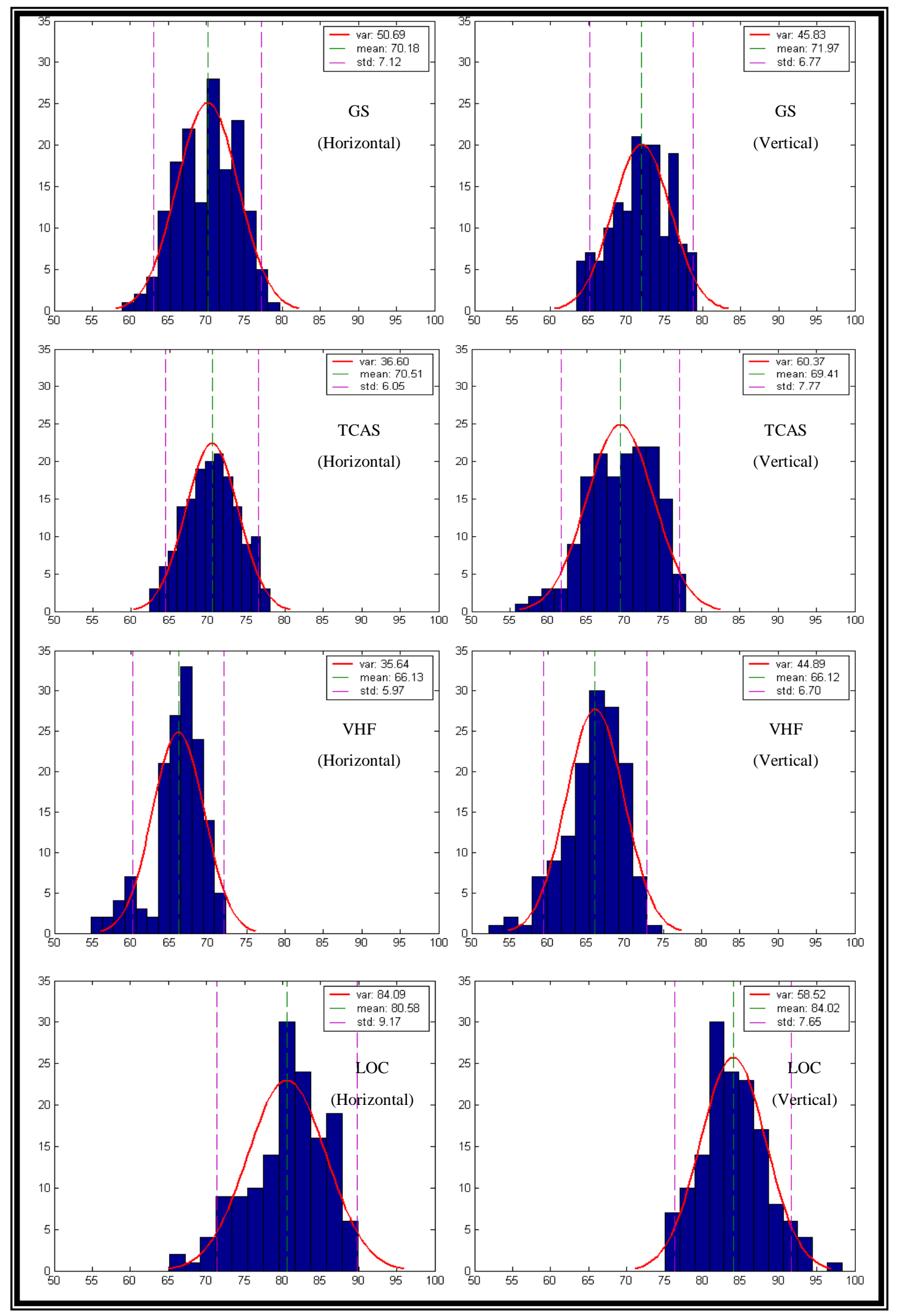

Figure 9: Normalized Plots with Mean, Variance and Standard Deviation for all four antennas, with horizontal and vertical polarization results 


\section{Data Confined to Window Locations Only:}

Taking window, seat and aisle IPL data on an entire airplane can be tedious, time consuming and expensive. Eight sets of measurements on each row of the aircraft are required, and there are 33 rows. In addition, measurements must be made at each of these points with the transmitter once in the vertical position, then in the horizontal: leading to 528 sets of measurements (on a B-737-200 series airplane). Most IPL data reported in the past includes only window measurements. Therefore, it is helpful to find any correlation between full sets of IPL data and window-only data, to better understand and use existing IPL data sets.

In this section, both graphical and statistical analysis is applied to determine whether the same characteristics and conclusions can be drawn from just the window data in comparison with the data taken on the entire aircraft.

\section{Graphical Comparison:}

Graphical plots were made for only the window locations of B-737 \#1989. These plots are included in Figure 10. After a careful comparison of these plots with the plots in Figures 4, 5, 6, and 7, several conclusions can be made:

The GS graphical plots show that horizontal polarization is dominant and the greatest coupling occurs in the front of the aircraft. These observations are the same for the entire airplane data set. The vertical polarization window plot does not fully reveal the minimum IPL values obtained away from the windows.

The TCAS graphical plots show that the vertical polarization is dominant and the greater coupling occurs at the front of the aircraft. These observations are the same for the entire airplane data set.

The VHF graphical plot results for the windows-only may be misleading, however. With a graphical plot of the entire airplane (Figure 6), it is clearly evident that optimal test antenna coupling is vertically polarized with the greatest coupling near the exit door. However, as seen in the window-only plot (Figure 10), the horizontal polarization appears to be dominant, and the window-only plot does not reveal that coupling rapidly diminishes when moving away from the window.

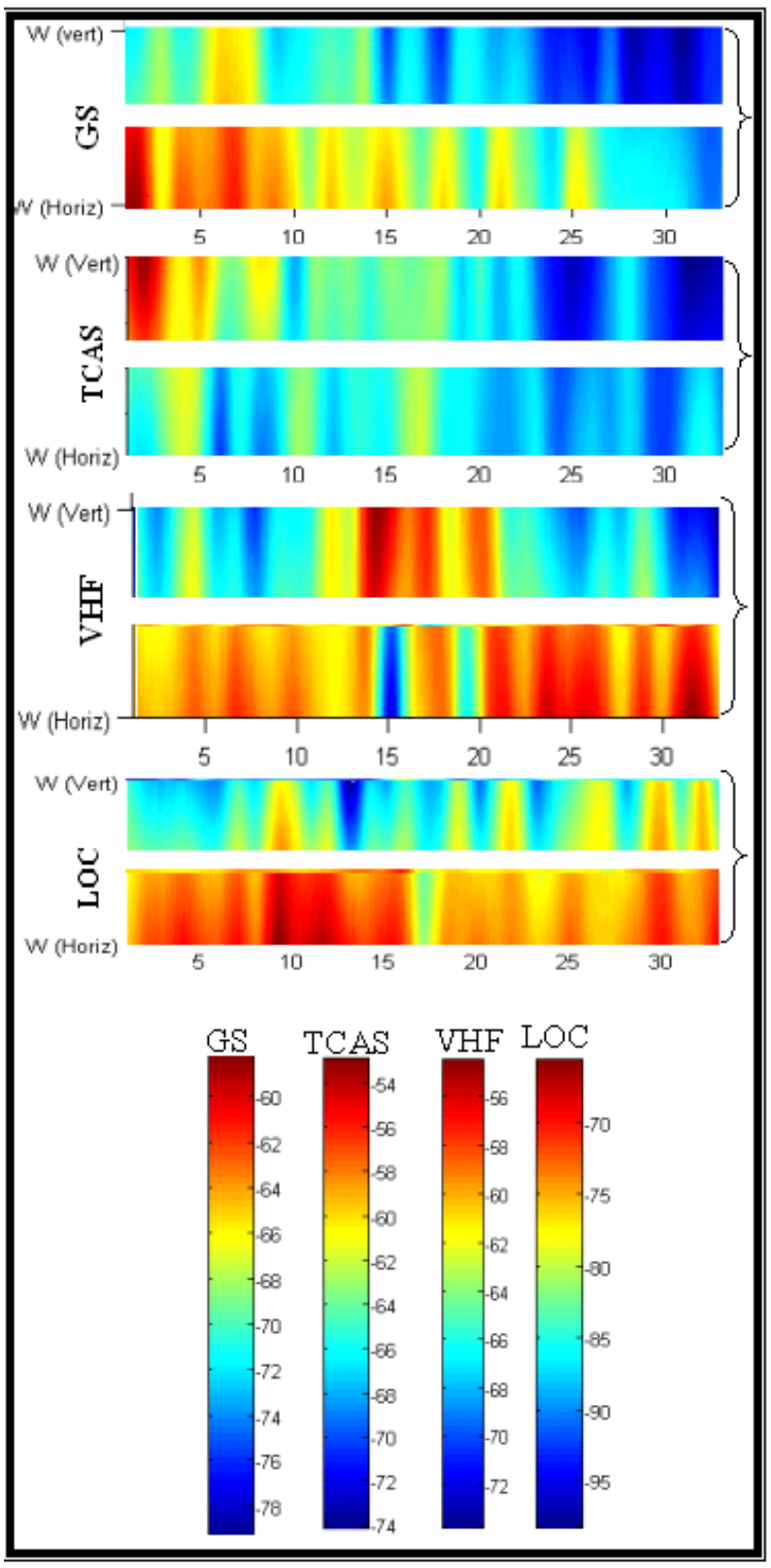

Figure 10: Window Representation for GS, TCAS, VHF and LOC (top), Color Maps for each type of Antenna for Reference (bottom)

The LOC graphical plots show that horizontal polarization is dominant and that comparable coupling levels occur throughout the aircraft. These observations are the same for the entire airplane data set. The vertical polarization window plot 
does not fully reveal the minimum IPL values obtained away from the windows.

In summary, the graphical comparison shows that taking data on the entire plane, although tedious and very time consuming, can reveal important insight into IPL coupling phenomena.

\section{Statistical Comparison:}

Similar to the graphical analysis, window data was separated and plotted individually for better comparison to the entire plane for a quantitative analysis. Please refer to Figures 11, 12, 13 and 14 for the measurement distribution plots of B737 \#1989 window data. These plots can be compared directly to those shown in Figure 9.

Table 1 includes a summary of all the minimums, maximums, standard deviations, mean and variance of each antenna. It also shows a comparison of data measured on full plane vs.

\begin{tabular}{|c|c|c|c|c|}
\hline & \multicolumn{2}{|c|}{ Full Data Set } & \multicolumn{2}{|c|}{ Window Data only } \\
\hline & Horiz ontal & Vertical & Horizontal & Vertical \\
\hline \multicolumn{5}{|l|}{ GS } \\
\hline Minimum & 59 & 64 & 59 & 66 \\
\hline Maximum & 80 & 79 & 74 & 79 \\
\hline Std. Deviation & 7.12 & 6.77 & 7.32 & 6.60 \\
\hline Mean & 70.18 & 71.97 & 67.13 & 73.07 \\
\hline Variance & 50.69 & 45.83 & 53.58 & 43.56 \\
\hline Dominant? & Yes & No & Yes & No \\
\hline \multicolumn{5}{|l|}{ TCAS } \\
\hline Minimum & 62 & 53 & 62 & 53 \\
\hline Maximum & 78 & 78 & 71 & 74 \\
\hline Std. Deviation & 6.05 & 7.77 & 3.82 & 9.35 \\
\hline Mean & 70.51 & 69.41 & 66.65 & 65.46 \\
\hline Variance & 36.60 & 60.37 & 14.97 & 87.42 \\
\hline Dominant? & No & Yes & No & Yes \\
\hline \multicolumn{5}{|l|}{ VHF } \\
\hline Minimum & 55 & 52 & 55 & 55 \\
\hline Maximum & 72 & 75 & 72 & 74 \\
\hline Std. Deviation & 5.98 & 6.70 & 5.94 & 8.35 \\
\hline Mean & 66.13 & 66.12 & 59.44 & 65.28 \\
\hline Variance & 35.64 & 44.89 & 35.10 & 69.72 \\
\hline Dominant? & No & Yes & No & Yes \\
\hline \multicolumn{5}{|l|}{ LOC } \\
\hline Minimum & 65 & 75 & 66 & 76 \\
\hline Maximum & 90 & 98 & 81 & 98 \\
\hline Std. Deviation & 9.17 & 7.65 & 5.45 & 8.54 \\
\hline Mean & 80.58 & 84.02 & 72.18 & 85.29 \\
\hline Variance & 84.09 & 58.52 & 29.38 & 72.93 \\
\hline Dominant? & Yes & No & No & Yes \\
\hline
\end{tabular}

window locations only. Comparison of measurement data for GS (Fig. 11 vs. Fig. 9) clearly shows that the minimum IPL values were measured at window locations. The mean values decrease 3.0 $\mathrm{dB}$ for horizontal polarization and $1.1 \mathrm{~dB}$ for vertical polarization, indicating a fairly good level of statistical comparability between whole aircraft vs. window-only IPL measurement data.

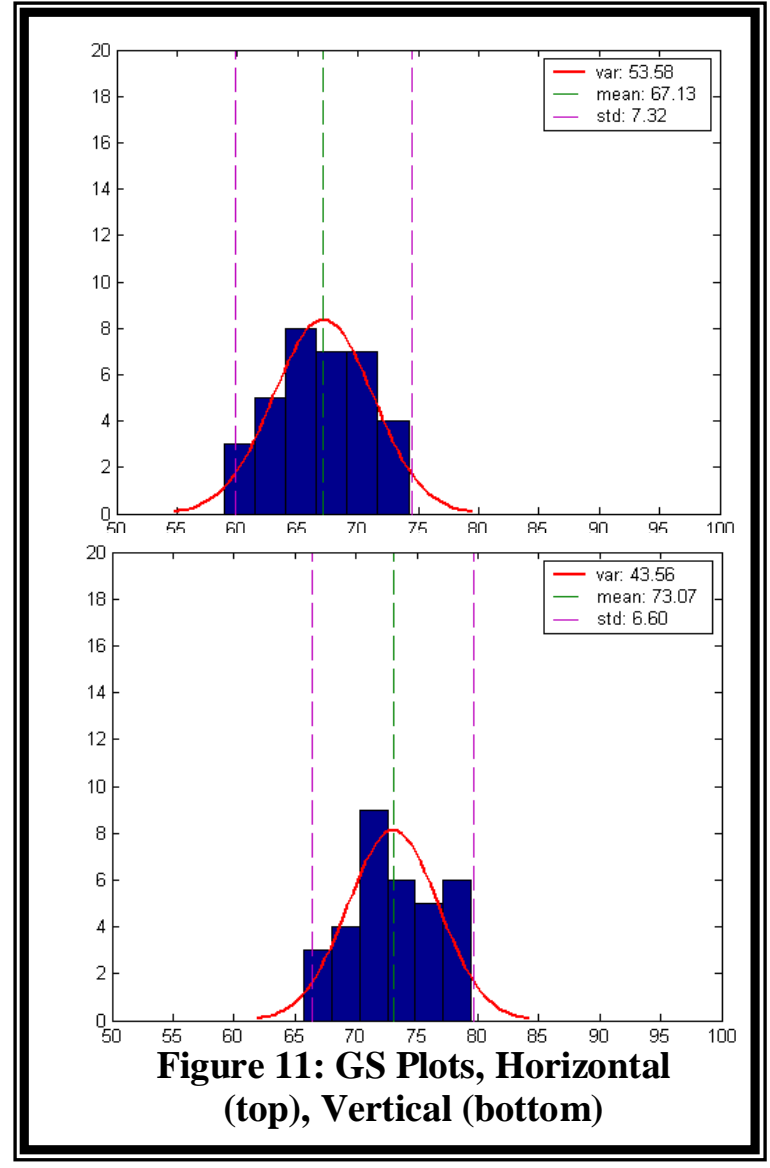

Comparison of measurement data for TCAS (Fig. 12 vs. Fig. 9) also shows that the minimum IPL values were measured at window locations. The mean values decrease $3.9 \mathrm{~dB}$ for horizontal polarization and $3.9 \mathrm{~dB}$ for vertical polarization, indicating a moderate statistical comparability between whole aircraft vs. window-only IPL measurement data.

Comparison of measurement data for VHF Com. (Fig. 13 vs. Fig. 9) shows that the minimum IPL value was not measured at a window location. An IPL value $3 \mathrm{~dB}$ less than the minimum window location was obtained when evaluating the whole aircraft data set. The mean values decrease $6.7 \mathrm{~dB}$ 


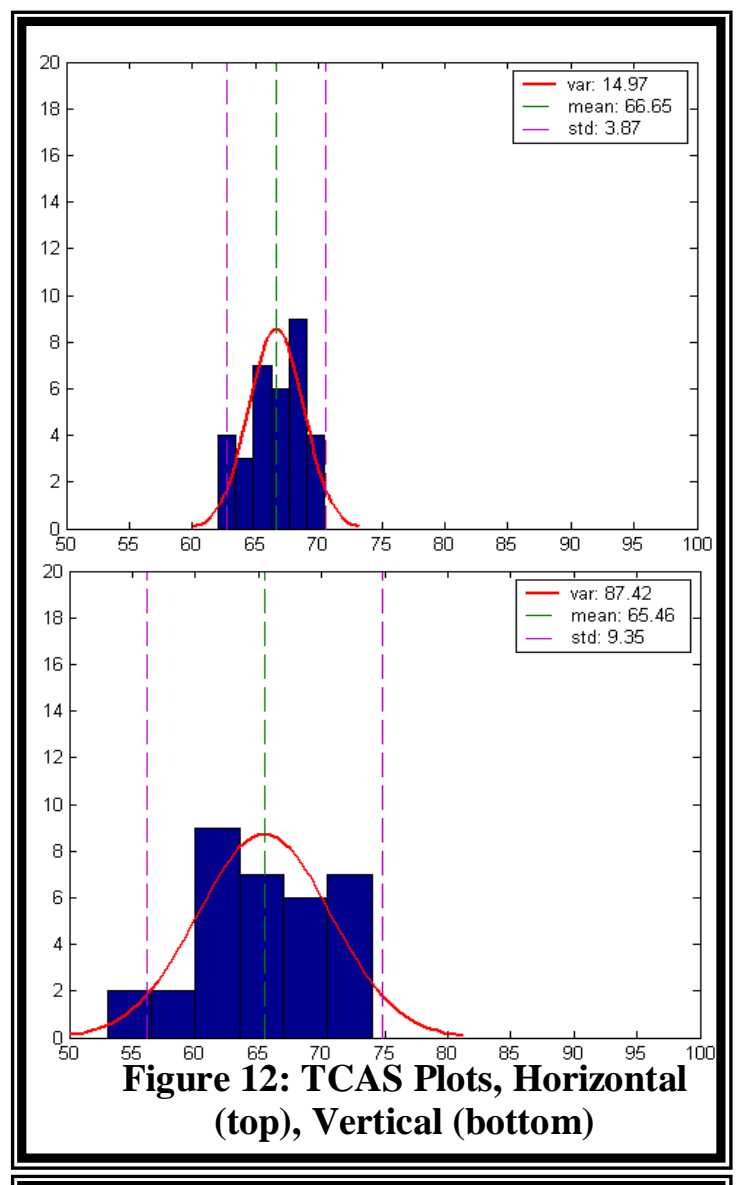

for horizontal polarization and $0.8 \mathrm{~dB}$ for vertical polarization, indication a good statistical comparability between whole aircraft vs. windowonly IPL measurement data, when considering vertical polarization, but not horizontal polarization.

Comparison of measurement data for LOC (Fig. 14 vs. Fig. 9) shows that the minimum IPL value was not measured at a window location. An IPL value $1 \mathrm{~dB}$ less than the minimum window location was obtained when evaluating the whole aircraft data set. The mean values decrease $8.4 \mathrm{~dB}$ for horizontal polarization and $1.3 \mathrm{~dB}$ for vertical polarization, indicating a good level of statistical comparability between whole aircraft vs. windowonly IPL measurement data for vertical polarization, but not horizontal polarization.

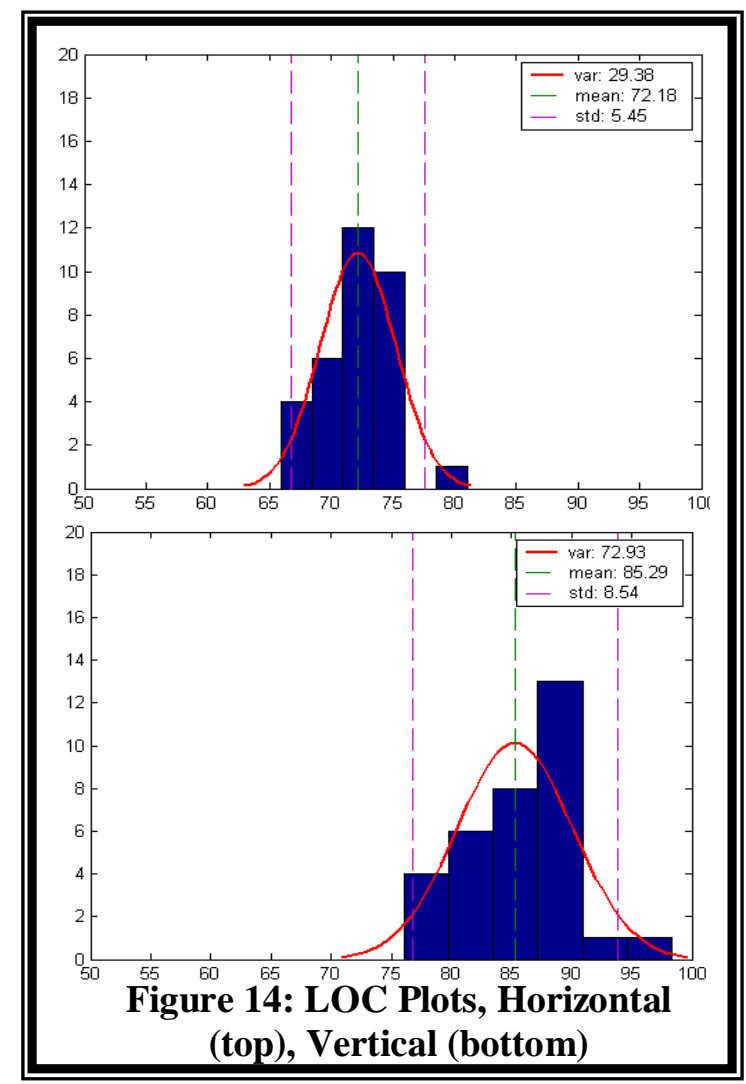

From the results above, it can be concluded that IPL data sets limited to window-only locations may slightly underestimate the minimum IPL in some cases (by up to $3 \mathrm{~dB}$ for the VHF Com. system, in this case). Also, statistical parameters are shown to vary considerably when comparing full-aircraft vs. window-only IPL data sets. Full sets of seat and aisle data need to be evaluated from 
other airplane types, so that general statistical relationships may be observed and predicted in comparing full data sets with window-only data sets.

\section{Comparison of Test Antennas: Dipole versus Biconical}

The VHF Com. data plotted in this paper previously utilized an ETS $3121 \mathrm{C}$ dipole Antenna, with antenna element lengths adjusted for optimum efficiency $(54.0 \mathrm{~cm}$ per element in the VHF Com. frequency band).
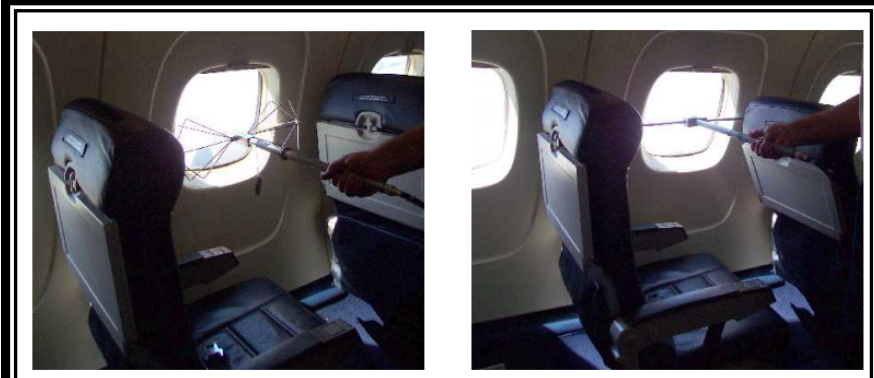

Figure 15: Biconical Antenna (left) and Dipole Antenna (right) in a horizontal testing position

It was hypothesized that since the dipole antenna is as long as three window spans, it may be likely that the data obtained is not applicable in real world applications. (It is very unlikely for a passenger to carry a PED large enough to span the length of three windows.) Therefore, it was proposed that similar IPL measurements be taken on the entire airplane, using a smaller antenna, to see if the IPL patterns change for the horizontal polarization.

A Schwarzbeck UBAA9114/BBVu9135 small biconical antenna was used to repeat the entire set of IPL measurements for the VHF Com. aircraft system. The Schwarzbeck small biconical antenna is much smaller than the ETS $3121 \mathrm{C}$ dipole antenna (44.4 cm total length), and covers only about one window span of an aircraft, resembling a real world PED more closely. Relative to the dipole antenna, the Schwarzbeck small biconical antenna has 10.7 $\mathrm{dB}$ less gain $(-10.7 \mathrm{~dB})$ in the VHF Com frequency band. This is primarily due to reflection loss because of its small electrical size in the VHF Com frequency band.

Figure 16 shows the MATLAB plot of the entire aircraft VHF Com IPL, when transmitting through the small biconical test antenna. The raw data was adjusted by $+10.7 \mathrm{~dB}$ to account for the relative gain of the small biconical antenna to the dipole antenna. This plot can be compared directly with the VHF Com plot shown in Figure 6.

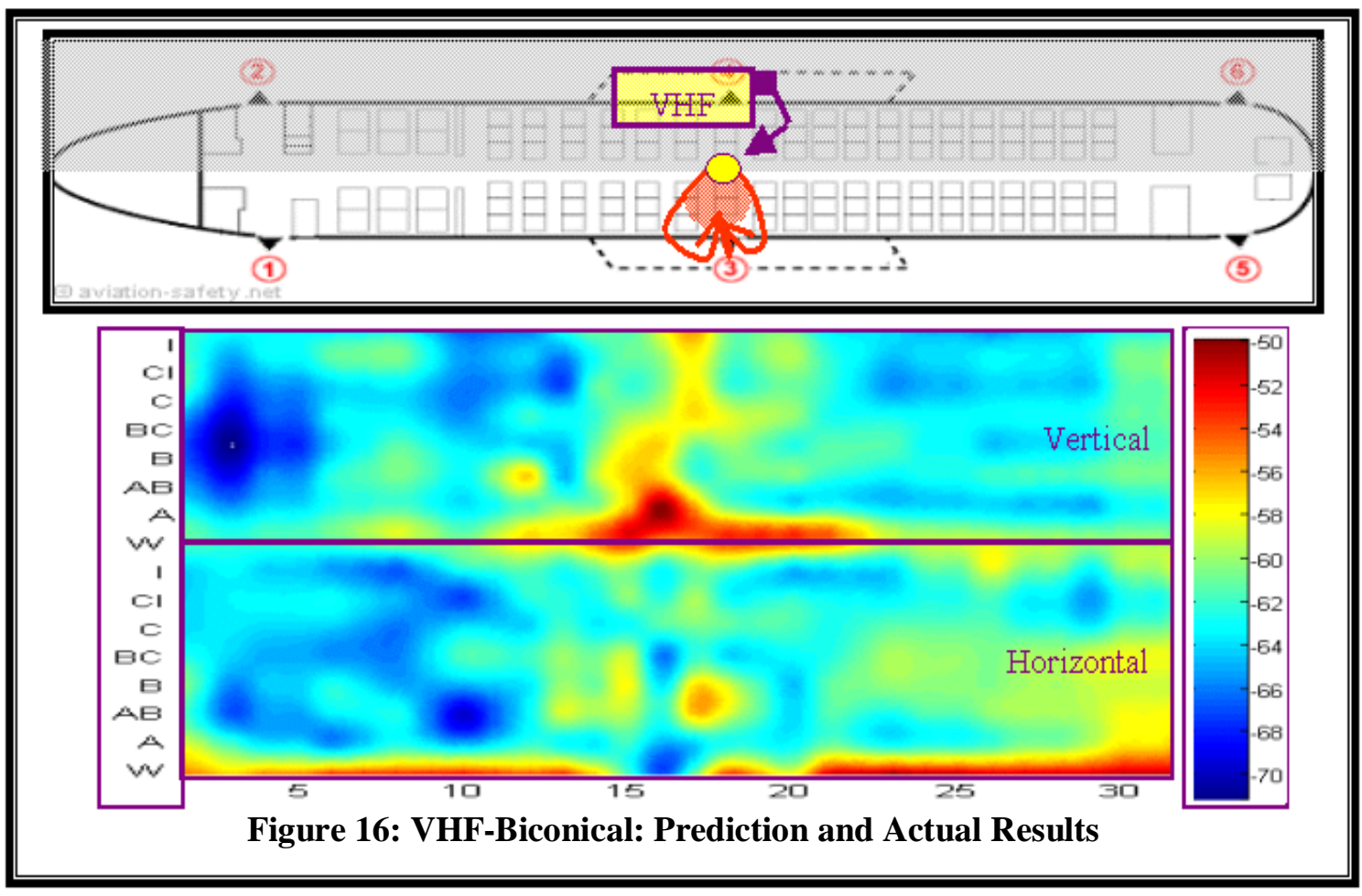


Surprisingly, there is almost no difference in the EMI patterns of the plots. The line of greater coupling near the windows of the horizontal polarized data is still present in the plots with biconical antenna, and in fact more pronounced. This indicates that the improved horizontal polarization coupling near windows is not an artifact of the IPL measurement process, but an actual physical phenomenon. The pattern near window 16 of high coupling in the vertical polarization is also very similar. Most importantly, application of the $-10.7 \mathrm{~dB}$ gain factor for the small biconical antenna results in a nearly identical IPL magnitude scale.

\section{Conclusion:}

This paper provides analysis of IPL data measured on out-of-service United Airlines B-737 airplanes. IPL propagation characteristics throughout an aircraft are graphically presented for four airplane radio receiver systems. The graphical data presentation clearly reveals particular PED locations where the threat due to Electromagnetic interference is greatest (ie. lowest IPL), and provides insight as to how PED orientation may play a major role in coupling due to polarization effects. In most cases, optimal PED coupling locations are shown to be predictable with knowledge of the airplane antenna location and polarization. However, in the case of one aircraft system (LOC), the actual IPL pattern clearly depends upon other factors.

To evaluate whether the IPL measurement process was repeatable, graphical IPL data plots were created from a different set of IPL data, measured on a different B-757-200 airplane, using different test equipment and different personnel. The graphical results are shown to be nearly identical for both airplanes, indicating that the measurement process was highly repeatable.

A statistical analysis of IPL data is provided. Measurement distribution plots were generated to clearly show the upper and lower limits of the IPL measurements, as well as their mean, variance, and standard deviation. Measurement distribution plots provide clear insight into the relative fraction of measurement locations responsible for the minimum IPL data. IPL mean, variance and standard deviation information is reported to allow comparison this IPL data from other sources on similar and different airplane types.

Graphical IPL data plots and statistical analysis were applied to a subset of window-only measurements, and compared with the wholeaircraft data set. The analysis clearly shows that IPL data sets limited to window-only locations may slightly underestimate the minimum IPL in some cases. Also, statistical parameters are shown to vary noticeably when comparing full-aircraft vs. window-only IPL data sets. Full sets of seat and aisle data need to be evaluated from other airplane types, so that general statistical relationships may be further documented in comparing full data sets with window-only data sets.

Finally, a comparison of different test antennas for IPL measurement was performed. A small biconical antenna was demonstrated to provide identical IPL measurement results to a much larger dipole antenna for the VHF radio frequency band. The small biconical antenna is significantly more rugged and easier to manipulate than a dipole antenna, and does not require element length adjustments for different frequency bands.

It is anticipated that this work will lead to more efficient IPL measurements and better understanding of IPL coupling phenomenon. Similar analysis of IPL data on additional airplane types may provide a basis for minimizing the amount of IPL data required for characterizing the PED threat to aircraft radios. Analysis of IPL data on different airplane types may also allow the development of predictive tools for maximizing IPL (thus minimizing the potential for PED EMI) by recommending configuration changes to aircraft.

\section{References}

[1] Fuller, Gerald. "B737-200 Path Loss Tests: Victorville, California." Mariposa, CA, March 2002, Task 1 report under NASA Contract L-16099

[2] T. X. Nguyen, S. V. Koppen, J. J. Ely, R. W. Williams, L. J. Smith, M. P. Salud, "Portable Wireless LAN Device and Two-Way Radio Threat Assessment for Aircraft Navigation Radios", NASA/TP-2003-212438, July 2003

[3] RTCA/DO-233, "Portable Electronic Devices Carried on Board Aircraft", August 20, 1996 